\title{
Attitudes of a group of Brazilian orthodontists towards the diagnosis and management of primary headache (migraine): an electronic-based survey
}

\author{
Reynaldo Leite MARTINS JUNIOR ${ }^{1}$, Florence de Carvalho KERBER ${ }^{2}$, Juliana STUGINSKI-BARBOSA ${ }^{3}$
}

\begin{abstract}
1- DDS, MSc, Professor of Orofacial Pain, Dental School, Univag-Centro Universitário Várzea Grande, MT, Brazil; Department of Dentistry, Mato Grosso Cancer Hospital, Cuiabá, MT, Brazil; Postgraduate Student in Orthodontics, Faisa/Sinodonto, Cuiabá, MT, Brazil.

2- DDS; Undergraduate medical student, Medical School, University of Cuiabá, Cuiabá, MT, Brazil.

3- DDS, Master's degree student in Neuroscience, Medical School of Ribeirão Preto, University of São Paulo, Ribeirão Preto, SP, Brazil.
\end{abstract}

Corresponding address: Reynaldo Leite Martins Jr. - Av. São Sebastião 2895 - 78045-305 - Bairro Goiabeiras - Cuiabá - MT - Brazil - e-mail: reynaldo@ terra.com.br

Received: September 30, 2009 - Modification: April 08, 2010 - Accepted: May 25, 2010

\section{ABSTRACT}

\begin{abstract}
bjectives: The aim of this study was to investigate the knowledge and attitudes of orthodontists in the diagnosis and management of migraine without aura. Material and Methods: Participants were dentists, recruited among members of the Brazilian Association of Orthodontics and Facial Orthopedics (ABOR). An e-mail was sent to all ABOR members, with a link to a website, especially prepared for this research. Dentists were presented to a report of a fictional patient fulfilling diagnostic criteria for a primary headache disorder, known as migraine without aura. Participants were asked to describe how they would relieve the patient's pain. Professional procedures were classified as "adequate" or "inadequate" according to the answers given. Results: 161 valid answers were received ( $18.8 \%$ response rate). Of them, $36 \%$ of the actions were considered to be "adequate" procedures, while 64\% were "inadequate". The results yielded 12 main procedures, based on common characteristics. Eighty-two orthodontists suggested orthodontic treatment with or without orthognathic surgery, and some suggested using stabilization appliances prior to the orthodontic treatment. Conclusions: The majority of participants proposed inadequate therapies, and $51 \%$ suggested orthodontic correction of occlusion, including orthognathic surgery. Educational activities on migraine should also target orthodontists.
\end{abstract}

Key words: Orthodontics. Dental occlusion. Migraine disorders. Facial pain. Temporomandibular joint disorders. Diagnostic errors.

\section{INTRODUCTION}

Dental occlusion is within the scope of many dental specialties, such as prosthetic dentistry and orthodontics. Besides temporomandibular disorders (TMD), anecdotal reports relate it as being an etiologic factor in a series of morbid conditions, such as, headaches, poor posture and lumbar disc prolapses $7,16,22,28$.

The International Classification of Headache Disorders (ICHD-II) categorizes headaches as primary, if not caused by another disorder, and secondary, if caused by another disorder. Thus, conditions such as aneurisms, tumors or trauma cause secondary headaches (headache is a symptom of the underlying medical illness), whereas the headaches of migraine are primary ones. However, it is important to underline that the term "primary" does not mean nonorganic, idiopathic or without a neurologic basis ${ }^{27}$.

Migraine is a well recognized condition, with specific evidence-based treatment protocol that did not include any kind of irreversible dental procedures as occlusal adjustment or orthodontics ${ }^{26}$.

Notwithstanding, in magazine articles to the general public, headache is often said to be caused by dental, occlusal and muscular problems. Indeed, two of the most important Brazilian weekly magazines published articles with statements about the relationship between poor dental occlusion and 
headache 23,29 . These magazines are read by over 4 million individuals. Misinformation of this nature has the potential to generate a huge demand for orthodontic therapies from individuals with migraine.

Accordingly, the aim of this study was to investigate the knowledge and attitudes of orthodontists regarding the diagnosis and management of a case of a primary headache, migraine without aura.

\section{MATERIAL AND METHODS}

Participants were dentists recruited among members of the Brazilian Association of Orthodontics and Dentofacial Orthopedics (ABOR). Of the 1,250 members, 1,206 made their e-mail addresses available and all of them were contacted with an invitation to participate in the research and a link to a website with an informed consent form and a fictional case report.

The case was of a 23 year-old woman, with throbbing pain for several years. Episodes lasted around $24 \mathrm{~h}$, recurring about twice a month. Pain was located on the orbital and periorbital regions, spreading to the zygomatic arch, always on the right side. The pain was accompanied by photophobia, phonophobia and nausea, and increased with physical activities. Attacks were severe enough for the patient to avoid routine activities. The case described fulfilled all the criteria for a primary headache disorder, known as migraine without aura.

The hypothetical patient had been seen by other specialists, who ruled out ophthalmological and otorhinolaryngological pathologies, rhinosinusitis or odontalgia. Previous treatments consisted of physical therapy, posture correction, hot packs and ice packs applications, transcutaneous electric nerve stimulation (TENS) and low-level laser therapy. None of them yielded satisfactory results.

Cranial computed tomography (CT), magnetic resonance imaging (MRI), and radiography of the teeth were normal. Clinical examination found excellent oral hygiene and no signs of periodontal disease. Occlusion exam demonstrated deep overbite and crossbite on the right side.

Two actions were requested: participants were asked to describe their therapeutic approach in order to relieve patient's pain; after that, they were inquired if the choice was based on what had been taught to them in the graduate or continuing education programs in Orthodontics. The answers were to be written in a maximum of 3 lines.

Participants could tell us if they would be interested in receiving the results of the research. Data were de-identified. Participant's therapeutic procedures were classified as "adequate" or "inadequate". Any procedure that postponed adequate treatment for migraine was considered "inadequate", even if it was a conservative and reversible procedure. Adequate procedures including referring the patient to a medical doctor or to dentists specialized in orofacial pain, for a throughout evaluation.

\section{RESULTS}

Of the 1,206 contacted individuals, 351 (29.10\%) e-mails were returned because of wrong electronic addresses. Of the 855 valid e-mails, 64 were answered on the first attempt; after 2 weeks, a second request was sent, yielding 63 answers. A third and final request yielded 41 responses. Of the 168 responses, one was a duplicate and 6 were in blank. Our sample consisted of 161 valid answers (18.3\% of valid e-mails).

The results were separated in 12 main procedures, based on common characteristics. They were then divided into two groups, one pooling "adequate" procedures $(n=58 ; 36 \%)$ (Table 1$)$, and the other pooling "inadequate" procedures $(n=102 ; 64 \%$ ) (Table 2). When palliative procedures (e.g. non-steroidal anti-inflammatory drugs - NSAIDs) were suggested until conducting orthodontic correction, only the later was considered, since it highlights the main treatment in the opinion of the specialist. Details of the proposed orthodontic or surgical techniques were not taken into consideration, since they were irrelevant to the study. The use of orthodontic apparatus with or without other procedures, such as orthognathic surgeries, was classified as "orthodontic correction of occlusion".

Thirty-three percent of the "adequate" and 58\% of the "inadequate" answers were based on what had been taught to the participants in the graduate or continuing education programs in Orthodontics course (Figure 1).

Table 1- Valid responses considered to be adequate

\begin{tabular}{lc}
\hline \multicolumn{1}{c}{ Responses } & $\mathbf{N}(\%)$ \\
\hline Suggests diagnosis and proposes referral to a neurologist or dentist specialized in TMD or orofacial pain & $9(5.6 \%)$ \\
Does not suggest diagnosis but proposes referral to a neurologist or dentist specialized in TMD or orofacial pain & $27(17.8 \%)$ \\
Diagnose migraine and proposes referral to a neurologist & $21(13 \%)$ \\
Diagnose migraine and proposes referral to a dentist specialized in TMD or orofacial pain & $1(0.6 \%)$ \\
Total & $58(36 \%)$ \\
\hline
\end{tabular}


Table 2- Valid responses with procedures considered to be inadequate

\begin{tabular}{lc}
\hline \multicolumn{1}{c}{ Responses } & N (\%) \\
\hline Stabilization appliance and orthodontic correction of occlusion. & $29(18 \%)$ \\
Stabilization appliance with diagnostic objectives, to be followed by orthodontic correction of occlusion & $19(11.8 \%)$ \\
\hline Orthodontic correction of occlusion & $34(21.1 \%)$ \\
Treatment with stabilization appliance & $7(4.4 \%)$ \\
Conservative treatment of TMD & $5(3.1 \%)$ \\
Prescription of non-steroidal anti-inflammatory drugs & $5(3.1 \%)$ \\
Further investigation & $3(1.9 \%)$ \\
Referral to an oral and maxillofacial surgeon) & $1(0.6 \%)$ \\
\hline Total & $103(64 \%)$ \\
\hline
\end{tabular}

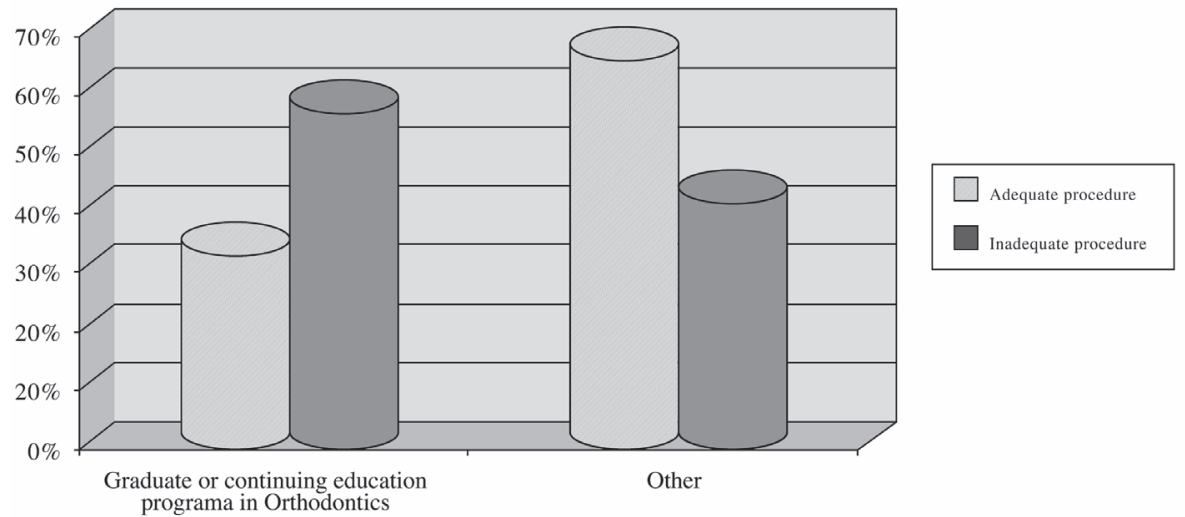

Figure 1- Relationship between proposed procedure and where the information was acquired

\section{DISCUSSION}

Web-based surveys have some advantages when compared to mailed surveys, such as lower costs, and the greater speed in obtaining responses ${ }^{3,25}$. Web-based surveys can be conducted through e-mail-based questions or by using webpages with questionnaires. The later is more efficient in protecting participant's confidentiality, possible getting higher response rates. Herein we created a web-page where individual responses were protected by individual user names and passwords ${ }^{11}$. This measure was also taken in order to avoid data contamination.

An important limitation of our study regards the external validation and the potential for sampling bias $^{2}$. According to the Brazilian Federal Dentistry Council data, there are 11,687 dentists with specialization in Orthodontics and/or Maxillary Functional Orthopedics in Brazil, but only 1,250 of them are members of the ABOR. Of them, most had their electronic addresses available, but less than $20 \%$ responded to the survey. It may be that professionals who are frequently users of internet were more likely to answer the survey. Furthermore, those who answered are probably more interested in the study subject ${ }^{10}$.
Nevertheless, there is no reason to believe that inclusion of non-members of the ABOR would yield to an increased frequency of proper approaches towards migraine. Indeed, if our results are biased, they are toward the opposite direction, since experts are more likely to engage in continued education. Open questions were chosen in order to avoid random choice of answers, as well as in order to more faithfully simulate a true appointment.

Due to the diversity of manifestations and different mechanisms of pain transmission, the differential diagnosis is crucial for the establishment of a successful management strategy ${ }^{4,5}$. Some dentists misdiagnosed most of frequent sources of orofacial pain (e.g. trigeminal neuralgia). If they had a basic education in differential diagnosis, they could refer the patient properly, allowing the establishment of a scientific based management strategy. Among those who diagnosed migraine and referred the patient to a headache specialist $(13 \%)$, three made an option to prescribe a triptan (class of drugs specifically used in the management of acute migraine crisis) to be used if needed, until the doctor's appointment. Referral to dentists with expertise in orofacial pain was considered as adequate, as they are more likely to detect patients in need of referral and to conduct the differential 
diagnosis.

One-hundred and two answers were classified as "inadequate conducts". Eighty-two orthodontists suggested orthodontic treatment with or without orthognathic surgery. Some of them would first use stabilization appliances as a therapeutic test. Since the placebo effect of bite-guard splints is well documented ${ }^{6}$, we classified as "inadequate", because an eventual improvement in the patient's condition due to unspecified factors in the treatment would lead the patient to a subsequent orthodontic treatment. It is worth mentioning, however, that $59 \%$ of the orthodontists participating in the study considered that poor occlusion explained the headaches.

We have discarded one case that properly diagnosed migraine and provided an adequate treatment according to literature data ${ }^{26}$. Although the treatment was correct, dentists are not allowed to treat migraine in Brazil.

Regarding the second question, most (58\%) participants who proposed an "inadequate" procedure stated that their answer was based on what had been taught to them in the graduate or continuing education programs in Orthodontics. Interestingly, the percentage was smaller in those who offered "adequate" procedures (33\%), and was lowest $(22 \%)$ in those who diagnosed migraine. The data clearly suggest flaws and specific needs for headache education targeting dentists.

The case presented was fully compatible with the diagnosis of migraine without aura ${ }^{27}$. This condition affects about 15 to $18 \%$ of women and 4 to $8 \%$ of men in North America, South America and Europe. Prevalence increases in both genders until the third decade of life and declines after that ${ }^{17}$. In Brazil, the prevalence of migraine is $20.9 \%$ in women and $9.3 \%$ in men, with a $2.2: 1$ female/ male ratio ${ }^{20}$. The peak of prevalence in women occurs between the ages of 25 to 55 years, the fertile period ${ }^{18}$. On the other hand, according to the American Academy of Orofacial Pain, TMD is a collective term that embraces a number of clinical problems involving the masticatory muscles, the temporomandibular joint (TMJ) and the associated structures, or both. It is the most important cause of non-dental-related pain in the orofacial region; it is a subclass of the musculoskeletal disorders ${ }^{8}$. The symptoms associated to TMD include pain or discomfort inside or around the ears, TMJ and/ or masticatory muscles, face, temples and neck. Patients may have limitations on mouth opening, and worsening of symptomatology with chewing ${ }^{19}$. Since the fictional patient had signs of malocclusion, we suppose that, by correcting this malocclusion, the clinician was attempting to relieve symptoms of TMD; nonetheless, the case did not present any evidence of masticatory dysfunction. Furthermore, the relation of TMD with poor dental occlusion is at least controversial. Since occlusion problems are so prevalent ${ }^{1}$, they are also frequent in those with TMD. The TMJ condyle position is very variable in TMD patients as well as in asymptomatic volunteers ${ }^{21}$. Finally, high rate of success with conservative treatment of TMD have been reported with therapies that do not modify the patterns of occlusion ${ }^{13,14}$ and even with placebo therapies ${ }^{12}$.

Several studies suggest that orthodontic treatment does not prevent $\operatorname{TMD}^{9,30}$. The same is true for occlusal adjustments ${ }^{15}$.

When data collection was finalized, participants received information on the results. Some participants questioned the "inadequacy" of orthodontic treatments for the case, since there would be no "proof" that occlusion "would not be responsible for the symptoms presented". In our point-of-view, this approach unfortunately excludes the unequivocal possibility of the migraine diagnosis, a well defined condition with specific diagnostic criteria ${ }^{27}$ and established treatment protocols ${ }^{26}$. Indeed the use of orthodontic correction for migraines should not be used until positive randomized controlled trials are published. The results of this survey seem to corroborate with the idea that most of specialized professionals (and not only Orthodontics specialists) are accustomed to apply the treatment they are used to do in their day-by-day activities, regardless of a proper diagnostic process. It is something like "to do the thing right, but not the right thing". Furthermore, the delay in properly diagnosing migraine may prolong suffering and expose patients to inadequate therapies, contributing to the discredit of health professionals, especially dentists, and increasing the risk of migraine chronification ${ }^{24}$.

\section{CONCLUSIONS}

The majority of the orthodontists misdiagnosed migraine and mistakenly suggested the use of orthodontic treatments, or other management strategies, usually applied in TMD cases, in a woman with migraine. Educational activities on migraine should also target orthodontists.

\section{REFERENCES}

1- Agerberg G, Sandström R. Frequency of occlusal interferences: a clinical study in teenagers and young adults. J Prosthet Dent. 1988;59:212-7.

2- Braithwaite D, Emery J, De Lusignan S, Sutton S. Using the Internet to conduct surveys of health professionals: a valid alternative? Fam Pract. 2003;20:545-51.

3- Cobanoglu C, Warde B, Moreo P. A comparison of mail, fax, and web-based survey methods. Int J Market Res. 2001;43:441-52. 4- Conti AC, Oltramari PV, Navarro RL, Almeida MR. Examination of temporomandibular disorders in the orthodontic patient: a clinical guide. J Appl Oral Sci. 2007;15:77-82. 
5- Conti PCR, Pertes RA, Heir GM, Nasri C, Cohen HV, Araújo CRP. Orofacial pain: basic mechanisms and implication for successful management. J Appl Oral Sci. 2003;11:1-7

6- Dao TT, Lavigne GJ, Charbonneau A, Feine JS, Lund JP. The efficacy of oral splints in the treatment of myofascial pain of the jaw muscles: a controlled clinical trial. Pain. 1994;56:85-94.

7- Davies S, Gray RM. The examination and recording of the occlusion: why and how. Br Dent J. 2001;191:291-6, 299-302.

8- De Leeuw R. Orofacial pain: guidelines for assessment, diagnosis, and management. Chicago: Quintessence Publishing Co.; 2008.

9- Egermark I, Carlsson GE, Magnusson T. A prospective long-term study of signs and symptoms of temporomandibular disorders in patients who received orthodontic treatment in childhood. Angle Orthod. 2005;75:645-50.

10- Eysenbach G, Wyatt J. Using the Internet for surveys and health research. J Med Internet Res. 2002;4:E13.

11- Fischbaker C, Chappel D, Edwards R, Summerton N. Health surveys via the internet: quick and dirty or rapid and robust? J R Soc Med. 2000;93:356-9.

12- Goodman P, Greene CS, Laskin DM. Response of patients with myofascial pain-dysfunction syndrome to mock equilibration. J Am Dent Assoc. 1976;92:755-8.

13- Greene CS. The etiology of temporomandibular disorders: implications for treatment. J Orofac Pain. 2001;15:93-105.

14- Kato MT, Kogawa EM, Santos CN, Conti PC. TENS and low-level laser therapy in the management of temporomandibular disorders. J Appl Oral Sci. 2006;14:130-5.

15- Koh H, Robinson PG. Occlusal adjustment for treating and preventing temporomandibular joint disorders. J Oral Rehabil. 2004;31:287-92.

16- Lambourne C, Lampasso J, Buchman WC Jr, Dunford R, McCall W. Malocclusion as a risk factor in the etiology of headaches in children and adolescents. Am J Orthod Dentofacial Orthop. 2007; 132:754-61.

17- Lipton RB, Bigal ME, Diamond M, Freitag F, Reed ML, Stewart WF; AMPP Advisory Group. Migraine prevalence, disease burden, and the need for preventive therapy. Neurology. 2007;68:343-9. 18- Lipton RB, Silberstein SD, Stewart WE. An update on the epidemiology of migraine. Headache. 1994;34:319-28.
19- Lobbezoo-Scholte AM, Lobbezoo F, Steenks MH, De Leeuw JR, Bosman F. Diagnostic subgroups of craniomandibular disorders. Part II: symptom profiles. J Orofac Pain. 1995;9:37-43.

20- Queiroz LP, Peres MFP, Piovesan EJ, Kowacs F, Ciciarelli MC, Souza JA, et al. A nationwide population-based study of migraine in Brazil. Cephalalgia. 2009;29:642-9.

21- Ren YF, Isberg A, Westesson PL. Condyle position in the temporomandibular joint. Comparison between asymptomatic volunteers with normal disk position and patients with disk displacement. Oral Surg Oral Med Oral Pathol Oral Radiol Endod. 1995; 80:101-7.

22- Rinchuse DJ, McMinn JT. Summary of evidence-based systematic reviews of temporomandibular disorders. Am J Orthod Dentofacial Orthop. 2006;130:715-20.

23- Rodrigues G. Da boca para o resto do corpo. Revista Isto é [serial on the internet]. [cited on $2010 \mathrm{Apr} 08$ ] 2007;1982. Available from: http://www.istoe.com.br/reportagens/4870DA+ $B O C A+P A R A+O+R E S T O+D O+C O R P O$ ?pathImagens $=\& p a t h=\& a c$ tualArea $=$ internalPage.

24- Scher AI, Midgette LA, Lipton RB. Risk factors for headache chronification. Headache. 2008;48:16-25.

25- Schleyer TK, Forrest JL. Methods for the design and administration of web-based surveys. J Am Med Inform Assoc. 2000;7:416-25.

26- Tfelt-Hansen P. A review of evidence-based medicine and meta-analytic reviews in migraine. Cephalalgia. 2006;26:1265-74. 27- The international Classification of Headache Disorders ( $2^{\text {nd }}$ Edition). Cephalalgia. 2004;24(Sp Issue 1):9-160.

28- Vélez AL, Restrepo CC, Peláez-Vargas A, Gallego GJ, Alvarez E, Tamayo $\mathrm{V}$, et al. Head posture and dental wear evaluation of bruxist children with primary teeth. J Oral Rehabil. 2007;34:663-70.

29- Vieira C. Enxaqueca. Revista Época [serial on the internet]. [cited on $2010 \mathrm{Apr}$ 08] 2003;293. Available from: http:// revistaepoca.globo.com/Revista/Epoca/0,_EMI41935-15223,00SAUDE+FITNESS.html.

30- Wadhwa L, Ultreja A, Tewari A. A study of clinical signs and symptoms of temporomandibular dysfunction in subjects normal occlusion, untreated, and treated malocclusions. Am J Orthod Dentofacial Orthop. 1993;103:54-61. 